\title{
Nonthermal support for the outer intracluster medium
}

\author{
A. Cavaliere ${ }^{1}$, A. Lapi ${ }^{1,2}$, and R. Fusco-Femiano ${ }^{3}$
}

\author{
1 Dip. Fisica, Univ. "Tor Vergata”, via Ricerca Scientifica 1, 00133 Roma, Italy \\ e-mail: lapi@roma2 .infn.it \\ 2 SISSA, via Bonomea 265, 34136 Trieste, Italy \\ 3 INAF-IASF, via Fosso del Cavaliere, 00133 Roma, Italy
}

Received 13 July 2010 / Accepted 18 October 2010

\begin{abstract}
We submit that nonthermalized support for the outer intracluster medium in relaxed galaxy clusters is provided by turbulence, which is driven by inflows of intergalactic gas across the virial accretion shocks. We expect this component to increase briskly during the cluster development for $z \lesssim 1 / 2$, owing to three factors. First, the accretion rates of gas and dark matter subside when they feed on the outer wings of the initial perturbations in the accelerating Universe. Second, the infall speeds decrease across the progressively shallower gravitational potential at the shock position. Third, the shocks eventually weaken and leave less thermal energy to feed the intracluster entropy, but relatively more bulk energy to drive turbulence into the outskirts. The overall outcome from these factors is physically modeled and analytically computed; thus we ascertain how these concur in setting the equilibrium of the outer intracluster medium, and predict how the observables in X-rays and $\mu$ waves are affected, so as to probe the development of outer turbulence over wide cluster samples. By the same token, we quantify the resulting negative bias to be expected in the total mass evaluated from X-ray measurements.
\end{abstract}

Key words. galaxies: clusters: intracluster medium - X-rays: galaxies: clusters - turbulence - methods: analytical

\section{Introduction}

In galaxy clusters the gravitational potential wells set by dark matter (DM) masses $M \sim 10^{15} M_{\odot}$ are filled out to the virial radius $R \sim$ Mpc by a hot thin medium at temperatures $k_{\mathrm{B}} T \sim$ several keVs, with central particle densities $n \sim 10^{-3} \mathrm{~cm}^{-3}$.

Such a medium constitutes an optimal electron-proton plasma (that we appropriately name IntraCluster Plasma, ICP), with its huge ratio of the thermal to the mean electrostatic energy $k_{\mathrm{B}} T / e^{2} n^{1 / 3} \sim 10^{12}$, and the relatedly large number $n \lambda_{\mathrm{D}}^{3}=$ $\left(k_{\mathrm{B}} T / 4 \pi e^{2} n^{1 / 3}\right)^{3 / 2} \sim 10^{16}$ of particles in the Debye cube. It emits copious X-ray powers $L_{\mathrm{X}} \propto n^{2} T^{1 / 2} R^{3} \sim 10^{45} \mathrm{erg} \mathrm{s}^{-1}$ via thermal bremsstrahlung, but with long radiative cooling times over most of the cluster's volume.

Thus on scales longer than the electro-proton mean free path, the ICP constitutes a quasi-neutral, simple fluid with 3 degrees of freedom in thermal equilibrium, and with effective particle mass $\mu m_{\mathrm{p}} \approx 0.6 m_{\mathrm{p}}$ in terms of the proton's $m_{\mathrm{p}}$. This affords precision modeling on the radial scale $r$ - to begin with - for the distributions of density $n(r)$ and temperature $T(r)$, so as to match the wealth of current and upcoming data concerning the emissions in X-rays (e.g., reviews by Snowden et al. 2008; Giacconi et al. 2009) and the strengths $y \propto n T_{\mathrm{e}}$ of the Sunyaev-Zel'dovich (1972) scattering in $\mu$ waves (e.g., Birkinshaw \& Lancaster 2007; Schäfer \& Bartelmann 2007).

In fact, simple yet precise modeling is provided by the SuperModel (SM; Cavaliere et al. 2009). This is based on the run of the ICP specific "entropy" (adiabat) $k \equiv k_{\mathrm{B}} T / n^{2 / 3}$ set by the processes for its production. The entropy is raised at the cluster centers owing to the energy discharged both by AGN outbursts (see Valageas \& Silk 1999; Wu et al. 2000; Cavaliere et al. 2002; McNamara \& Nulsen 2007) and by deep mergers (see McCarthy et al. 2007; Markevitch \& Vikhlinin 2007) often followed by inner sloshing (see ZuHone et al. 2010). At the other end, much entropy is continuously produced at the virial boundary. There the ICP is shocked by the supersonic gravitational inflow of gas accreted from the environment along with the DM (see Tozzi \& Norman 2001; Voit 2005; Lapi et al. 2005), and is adiabatically stratified into the DM potential well.

These physical processes concur to create ICP entropy distributions with spherically averaged profiles $k(r)=k_{\mathrm{c}}+\left(k_{R}-\right.$ $\left.k_{\mathrm{c}}\right)(r / R)^{a}$. These comprise a central floor $k_{\mathrm{c}} \approx 10-100 \mathrm{keV} \mathrm{cm}^{2}$ and an outer ramp with slope around $a \approx 1$, rising to join the boundary values $k_{R} \sim$ some $10^{3} \mathrm{keV} \mathrm{cm}^{2}$. Such values and shapes are consistent with recent observational analyses by Cavagnolo et al. (2009) and Pratt et al. (2010) out to $r \approx R / 2^{1}$.

The ensuing gradient of the thermal pressure $p(r) \propto$ $k(r) n^{5 / 3}(r)$ is used in the SM to balance the DM gravitational pull $-G M(<r) / r^{2}$ and sustain hydrostatic equilibrium (HE) out to the virial boundary. The $\mathrm{HE}$ equation may be written as $\mathrm{d} \ln T / \mathrm{d} \ln r=3 a / 5-2 b / 5$ in terms of the entropy slope, $a(r) \equiv \mathrm{d} \ln k / \mathrm{d} \ln r$, and of the potential to thermal energy ratio, $b(r) \equiv \mu m_{\mathrm{p}} v_{\mathrm{c}}^{2} / k_{\mathrm{B}} T$ with $v_{\mathrm{c}}^{2} \equiv G M(<r) / r$. From this, we directly derive the temperature profile (see Cavaliere et al. 2009, their Eq. (7))

$$
\frac{T(r)}{T_{R}}=\left[\frac{k(r)}{k_{R}}\right]^{3 / 5}\left\{1+\frac{2}{5} b_{R} \int_{r}^{R} \frac{\mathrm{d} x}{x} \frac{v_{\mathrm{c}}^{2}(x)}{v_{R}^{2}}\left[\frac{k_{R}}{k(x)}\right]^{3 / 5}\right\}
$$

in terms of the entropy run $k(r)$ and the boundary values at $r=R$. The density follows $n(r)=\left[k_{\mathrm{B}} T(r) / k(r)\right]^{3 / 2}$, so that $T(r)$ and $n(r)$ are linked, rather than independently rendered with multiparametric expressions as in other approaches. The X-ray and

${ }^{1} R / 2 \approx R_{500} \approx 2 R_{200} / 3$ holds in terms of the radii inside which the average DM overdensity relative to the critical universe amounts to 500 and 200 , respectively. 
SZ observables are readily derived from $T(r)$ and $n(r)$ and compared with data.

In preparation for the developments given below, we stress that the few parameters specifying $k(r)$ are enough for the SM to provide remarkably good fits to the detailed X-ray data on surface brightness and on temperature profiles of many clusters (see Fusco-Femiano et al. 2009). These include central temperature profiles of both main classes identified by Molendi \& Pizzolato (2001): the cool-cored CCs with a central dip and the centrally flat, non-cool-cored NCCs. The SM intrinsically links these morphologies to low or high entropy levels of $k_{\mathrm{c}} \sim 10^{1}$ or $\sim 10^{2} \mathrm{keV} \mathrm{cm}^{2}$, respectively, and they are conceivably imprinted by energy inputs from central AGN outbursts or from deep mergers (Cavaliere et al. 2009; Fusco-Femiano et al. 2009).

The SM also covers diverse outer behaviors, including cases where the entropy production decreases and its slope $a$ abates (see Lapi et al. 2010, and data references therein), to the effect of producing steep temperature profiles. The interested reader may try for her/himself other clusters by using the fast SM algorithm made available at the website http://people.sissa.it/ lapi/Supermodel/. Here we pursue another consequence of the diminishing entropy production, namely, turbulence arising in the outskirts of relaxed CC clusters.

Several observations (in particular with the Suzaku satellite, see George et al. 2009; Bautz et al. 2009; Hoshino et al. 2010; Kawaharada et al. 2010) support the notion that HE may be contributed by nonthermalized, turbulent motions occurring on scales of several $10^{2} \mathrm{kpc}$ inwards of $R$. On the other hand, several simulations resolve a variety of shocks in and around clusters (e.g., Ricker \& Sarazin 2001; Markevitch \& Vikhlinin 2007; Skillman et al. 2008; Vazza et al. 2010).

We focus on the accretion shocks that originate at the virial boundary from inflows of gas preheated at temperatures $10^{6} \mathrm{~K}$ by sources like outer stars and AGNs or by hydrodynamical processes like shocks around filaments. These two pieces of information led us to investigate whether the physics of the virial accretion shocks indeed requires turbulence to also develop in the outskirts of relaxed clusters under the smooth inflows that prevail there (Fakhouri et al. 2010; Genel et al. 2010; Wang et al. 2010).

\section{Virial accretion shocks: entropy and turbulence}

The key physical agent consists in the residual bulk flows downstream of the virial accretion shocks. The latter actually form a complex network ("shock layer", see Lapi et al. 2005) modulated to different strengths by the filamentary structure of their environment. Shock curvature leading to baroclinic instabilities and vortical flows, and/or sheared inflows inevitably arise as pioneered in the context of cosmic structures by Doroshkevich (1973) and Binney (1974), and recently demonstrated by several hydro-simulations (see Iapichino \& Niemeyer 2008; Ryu et al. 2008; Lau et al. 2009; Paul et al. 2010).

\subsection{Physics of virial accretion shocks}

Boundary shocks of high average strength arise in conditions of intense inflows of outer gas with supersonic speed $v_{1}$ corresponding to Mach numbers $\mathcal{M}^{2} \equiv v_{1}^{2} / c_{1}^{2} \sim 10^{2}$ relative to the outer sound speed $c_{1} \equiv\left(5 k_{\mathrm{B}} T_{1} / 3 \mu m_{\mathrm{p}}\right)^{1 / 2}$. These shocks effectively thermalize the inflows to produce postshock temperatures close to the ceiling $k_{\mathrm{B}} T_{2} \sim \mu m_{\mathrm{p}} v_{1}^{2} / 3$ that would mean full conversion of the infall $m_{\mathrm{p}} v_{1}^{2} / 2$ to thermalized energy $3 k_{\mathrm{B}} T / 2 \mu$ per

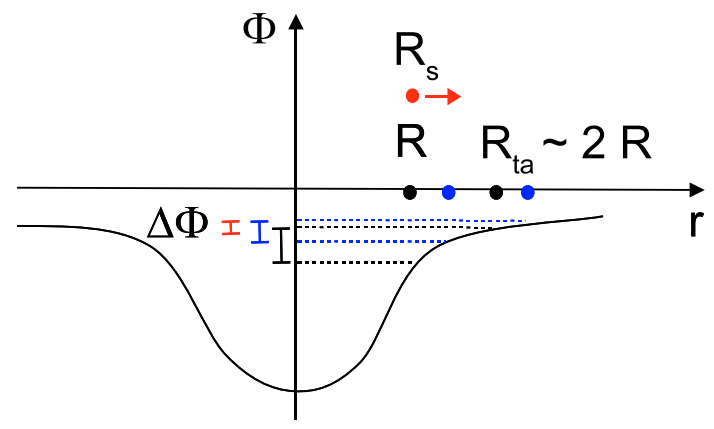

Fig. 1. The gravitational potential governing gas infall. As the DM outskirts develop (see Sect. 2), both the virial $R$ and the turnaround radius $R_{\mathrm{ta}}$ shift outwards, retaining the ratio $R_{\mathrm{ta}} / R \approx 2$. Meanwhile, the outer potential becomes shallower and $\Delta \Phi$ lower from the value marked in black to that in blue. The shock position $R_{\mathrm{S}}$ slowly outgrows $R$, lowering the drop to the value marked in red.

electron-proton pair. But even strong shocks hovering at $R_{\mathrm{S}} \approx R$ leave some residual postshock bulk flows with speed $v_{2} \approx v_{1} / 4$, see Lapi et al. (2005). These correspond to a kinetic energy ratio $v_{2}^{2} / v_{1}^{2} \approx 6.3 \%$.

Such a ratio is bound to grow, however, during the outskirts development. On the DM side, the outskirts develop inside out by secular accretion after the early central collapse (see Lapi \& Cavaliere 2009; Wang et al. 2010, and references therein). Such a trailing accretion feeds scantily on the outer, declining wings of the initial DM density perturbation that develops into a cluster, and is further impaired by the cosmic expansion accelerated by the dark energy (cf. Komatsu et al. 2010). In these conditions, the inflows will peter out, shock thermalization will be reduced, and eventually the shock themselves weaken, leaving postshock bulk energies enhanced well above the ratio $6.3 \%$.

To quantify the issue, we describe the perturbation shape in terms of the effective power law $\delta M / M \propto M^{-\epsilon}$ that modulates the mass excess $\delta M$ accreting onto the current mass $M$; in particular, low values of the shape parameter $\epsilon \lessgtr 1$ apply to the perturbation body, but $\epsilon$ grows larger for the outskirts (see $\mathrm{Lu}$ et al. 2006). A shell $\delta M$ will collapse on top of $M$ when $\delta M / M$ attains the critical threshold $1.69 D^{-1}(t)$ in terms of the linear growth factor $D(t)$, so the parameter $\epsilon$ also modulates the average mass growth reading $M(t) \propto D^{1 / \epsilon} \propto t^{d / \epsilon}$, with the growth factor represented by the power law $D(t) \propto t^{d}$ in terms of the exponent $d$. The latter decreases from $2 / 3$ to $1 / 2$ as the redshift lowers from values $z \gtrsim 1$ to $z \lesssim 1 / 2$, cf. Weinberg (2008). Thus the outskirts develop at accretion rates $\dot{M} / M \approx d / \epsilon t$ that lower as $\epsilon$ takes on values exceeding 1 in the perturbation wings (and formally diverging in voids), and as $d$ decreases to $1 / 2$ at late cosmic times.

On the ICP side, the outer gas will accrete with a lower infall speed $v_{1}$. As illustrated in Fig. 1 , it is set to $v_{1}^{2}=2 \Delta \Phi$ by the outer gravitational potential drop $\Delta \Phi \equiv \int_{R}^{R_{\mathrm{ta}}} \mathrm{d} r G \delta M / r^{2}$. This is experienced by successive shells of DM and gas that - after an initial expansion - turn around at the radius $R_{\mathrm{ta}} \approx 2 R$ to begin their infall toward the shock at $R_{\mathrm{S}} \approx R$. Thus the potential drop $\Delta \phi \equiv \Delta \Phi / v_{R}^{2}$ (normalized to the circular velocity $v_{R}^{2}=G M / R$ at $r=R$ ) reads as

$\Delta \phi=\frac{1-\left(R / R_{\mathrm{ta}}\right)^{3 \epsilon-2}}{3 \epsilon-2}$.

This is seen (cf. Fig. 1) to become shallower during the outskirt's development as $\epsilon$ exceeds 1 , after which the approximation $\Delta \phi \approx$ $(3 \epsilon-2)^{-1} \approx(3 \epsilon)^{-1}$ applies. 
Actually, the shock position $R_{\mathrm{S}}$ slowly outgrows the virial $R$ to approach $R_{\mathrm{ta}}$, and this yields an even lower effective potential drop (see Voit et al. 2003; Lapi et al. 2005). In fact, it can be shown that for $\epsilon>1$ the shock position may be approximated as $R_{\mathrm{S}} \approx 2 R\left(1-4 \epsilon^{-2}\right)$, see Lapi et al. (2010); so from Eq. (2) we obtain $\Delta \phi \approx 4 \epsilon^{-2}$.

The direct and indirect effects of $\dot{M}$ dwindling combine into the accretion rate scaling $\dot{M} \propto v_{1} M / R \propto v_{1}^{3} / \Delta \phi$, after which the infall speed follows

$v_{1} \propto \dot{M}^{1 / 3}(\Delta \phi)^{1 / 3}$.

This is indeed reduced strongly during the late development of the outskirts when both $\dot{M} \propto d / \epsilon$ subsides and $\Delta \phi \propto \epsilon^{-2}$ lowers, so that the overall scaling $v_{1} \propto d^{1 / 3} / \epsilon$ applies for $\epsilon>1$; specifically, as $\epsilon$ increases from 1 to 1.5 and then to 3 , the prevailing Mach numbers decline from $\mathcal{M}^{2} \approx 10$ to 6 and then to 3 .

These values are consistent with the Mach number distributions at low $z$ sliced for flows of preheated gas into the cluster, as found by numerical simulations (e.g., Ryu et al. 2003; Skillman et al. 2008, see their Fig. 6 and Sect. 4; see also Vazza et al. 2010).

\subsection{Weakening shocks and entropy demise}

With $v_{1}$ lowering toward transonic values, the shock strength will eventually weaken. We recall (see Appendix B in Lapi et al. 2005) that the Rankine-Hugoniot conservation conditions for a standing shock give the postshock temperatures and densities in the form

$\frac{T_{2}}{T_{1}}=\frac{7}{8}+\frac{5}{16} \mathcal{M}^{2}-\frac{3}{16} \frac{1}{\mathcal{M}^{2}} \quad, \quad \frac{n_{2}}{n_{1}}=\frac{4 \mathcal{M}^{2}}{3+\mathcal{M}^{2}}$.

As the Mach number decreases from values $\mathcal{M}^{2}>3$ (defining strong shocks) to transonic $\mathcal{M}^{2} \approx 1$, the temperature ranges widely from $k_{\mathrm{B}} T_{2} \simeq 3 \mu m_{\mathrm{p}} v_{1}^{2} / 16$ to $T_{2} \simeq T_{1}$, while the density varies mildly from $n_{2} \simeq 4 n_{1}$ to $n_{2} \simeq n_{1}$. Correspondingly, the shock-generated entropy $k_{2} \equiv k_{\mathrm{B}} T_{2} / n_{2}^{2 / 3}$ drops from values $5 \mathcal{M}^{2} k_{1} / 4^{2 / 3} 16 \sim$ some $10^{3} \mathrm{keV} \mathrm{cm}^{2}$ typical of strong shocks to intergalactic values $k_{1}=k_{\mathrm{B}} T_{1} / n_{1}^{2 / 3} \lesssim 10^{2} \mathrm{keV} \mathrm{cm}^{2}$.

By the same token, the entropy outer ramp will abate. Its slope at the boundary has been derived by Cavaliere et al. (2009) from the jumps at the shock and the adjoining HE recalled in Sect. 1, to read as

$a_{R}=\frac{\mathcal{A}}{2}\left(5-b_{R}\right)$.

Here, $\mathcal{A}=4(1+\epsilon / d) /[5+2(1+\epsilon / d)] \approx 1$ applies as long as $\epsilon \approx 1$ and $d \approx 2 / 3$ hold.

However, the main dependence of $a_{R}$ on $\epsilon$ and $d$ is encased in $b_{R} \equiv \mu m_{\mathrm{p}} v_{R}^{2} / k_{\mathrm{B}} T_{R}$ through the boundary temperature. We have just seen that $T_{2} \propto v_{1}^{2} \propto v_{R}^{2} \Delta \phi$ holds as long as strong shock conditions apply. If so, $b_{R} \propto 1 / \Delta \phi \propto \epsilon^{2}$ would rise fast as $\epsilon$ exceeds 1 ; but $v_{1}^{2} \propto d^{2 / 3} / \epsilon^{2}$ is meanwhile reduced and the virial accretion shocks weakened, slowing down the net growth of $b_{R}$. The overall result is that $b_{R}$ grows from the standard value 2.7 to about 5 and then to about 8 while $\epsilon$ increases from 1 to 1.5 and then to 3 .

After Eq. (5), increasing values of $b_{R}$ cause $a_{R}$ to decrease, and imply progressive saturation and even a decline in the entropy produced at the boundary. Specifically, as $\epsilon$ increases from 1 to 1.5 and then to 3 , the entropy slope $a_{R}$ goes from the standard value 1.1 to about 0 and then down to negative values around -2 .
To tackle the issue, recall that, while the cluster outskirts develop to the currently observed radius $R$, the ICP is adiabatically compressed and settles into the DM potential well. Then the specific entropy $k(r)$ stratifies shell by shell, leading to a running slope $a(r)=a_{R}$ that retains the sequence of the values set at the time of deposition (see Tozzi \& Norman 2001; Lapi et al. 2005). As a result, on moving out from the early cluster body to the outskirts currently building up by secular accretion, the whole slope $a(r)$ of the outer ramp decreases, and $k(r) \propto r^{a(r)}$ flattens or even bends over.

To describe this behavior, in Lapi et al. (2010) we used an entropy slope $a(r)=a-a^{\prime}\left(r-r_{b}\right)$ smoothly decreasing from the body value $a \approx 1.1$. We found a value $a \approx 0$ at $r \approx R / 2$ and values $a \approx-2$ at $R$ (as illustrated in Fig. 1 of Lapi et al. 2010), consistently with the data by Bautz et al. (2009) and George et al. (2009).

\subsection{Onset of turbulence}

Here we show that such an entropy demise due to $\dot{M}$ dwindling will arise together with the onset of turbulence triggered by shock weakening. In fact, the latter causes the postshock speeds,

$\frac{v_{2}}{v_{1}}=\frac{n_{1}}{n_{2}}=\frac{1}{4}+\frac{3}{4} \frac{1}{\mathcal{M}^{2}}$

to grow from values around $1 / 4$ and approach 1 . With $v_{1} \propto \epsilon^{-1}$ lowering sharply while $c_{1}$ varies as $(1+z)$ or less, the kinetic energy ratio is thus enhanced relative to the strong shock value $6.3 \%$, see Sect. 2.1. For example, as $\epsilon$ grows from 1 to 1.5 and then to $3-5$, the ratio $v_{2}^{2} / v_{1}^{2}$ increases from $10 \%$ to $14 \%$ and then to $25-39 \%$. The ratio $v_{2}^{2} / c_{2}^{2}$ of the residual bulk to the sound's speed past the shock also increases for decreasing $\mathcal{M}^{2}$; in particular, it goes from $25 \%$ to $50 \%$ as $\mathcal{M}^{2}$ ranges from 1 to 3-5. On average, for a CC cluster we find the condition $\mathcal{M}^{2} \propto d^{1 / 3} /\langle\epsilon(z)\rangle(1+z) \lesssim 3$ for shock weakening to be met at redshifts $z \lesssim 0.3$, on using for the average $\langle\epsilon\rangle$ the values given by Lapi \& Cavaliere (2009) in their Fig. 6. In a nutshell, combining Eqs. (4) and (6) yields an inverse relation of $v_{2}^{2} / v_{1}^{2}$ with $k_{2} / k_{1}$.

These postshock flows provide bounds to the energy level of subsonic turbulent motions that is driven by smooth accretion in relaxed clusters. Minor, intermittent, and localized contributions may be added by the complementary clumpy component of the accretion, recently re-calibrated to less than $30 \%$ in the outskirts of relaxed halos (see Fakhouri et al. 2010; Genel et al. 2010; Wang et al. 2010). Our bounds actually constitute fair estimates of the amplitudes of outer turbulent energy at $r \sim R$, as shown by similar values obtained both observationally (see Mahdavi et al. 2008; Zhang et al. 2010) and in many numerical simulations from Evrard (1990) to Lau et al. (2009).

\section{Modeling the turbulent support}

Turbulent motions start at the virial radius $R$ with comparable coherence lengths $L \sim R / 2$, set in relaxed CC clusters by the pressure scale height or by shock segmentation (see Iapichino \& Niemeyer 2008; Ryu et al. 2008; Pfrommer \& Jones 2010; Valdarnini 2010; Vazza et al. 2010). Then they fragment downstream into a dispersive cascade over the "inertial range", to sizes $\ell$ where dissipation begins after the classic picture by Kolmogorov (1941), Obukhov (1941), and Monin \& Yaglom (1965). In the ICP context the dissipation scale (equivalently to the classic Reynolds' scaling) writes as $\ell \sim$ $\left(c_{2} / \tilde{v}\right)^{3 / 4} \lambda_{\mathrm{pp}}\left(L / \lambda_{\mathrm{pp}}\right)^{1 / 4}$ in terms of the ion collisional mean free 
path $\lambda_{\mathrm{pp}}$ and of the ratio $\tilde{v} / c_{2}$ of the turbulent rms speed to the sound's, see Inogamov \& Sunyaev (2003). For subsonic turbulence with $\tilde{v} / c_{2} \lesssim 1 / 3$ (see direct observational bounds by Schücker et al. 2004; Sanders et al. 2010, and references therein) the relevant scale $\ell$ somewhat exceeds $\lambda_{\mathrm{pp}} \sim 10^{2} \mathrm{kpc}$.

In the presence of outer magnetic fields $B \lesssim 10^{-1} \mu \mathrm{G}$ (see Bonafede et al. 2010; Pfrommer \& Jones 2010; also Ryu et al. 2008), the key - if quantitatively debated - feature is their degree of tangling; then the effective mean free path is provided by the coherence scale of the field, which is somewhat larger than $\lambda_{\mathrm{pp}}$ (see discussions by Narayan \& Medvedev 2001; Inogamov \& Sunyaev 2003; Govoni et al. 2006; Brunetti \& Lazarian 2007).

Clearly the above estimates provide useful guidelines, but need theoretical modeling and observational probing. Both issues are addressed next. Since turbulent motions contribute to the pressure (see Landau \& Lifshitz 1959) to sustain HE, our modeling focuses on the ratio $\delta(r) \equiv p_{\text {nth }} / p_{\text {th }}$ of turbulent to thermal pressure (or, equivalently, on the ratio $\delta /(1+\delta)$ of turbulent to total pressure), with radial shape decaying on the scale $\ell$ from the boundary value $\delta_{R}$.

The total pressure is conveniently and generally written as $p_{\text {th }}(r)[1+\delta(r)]$, while the thermal component is still expressed as $p_{\text {th }}(r) \propto k(r) n^{5 / 3}(r)$. With this addition, we proceed by solving the HE equation just along the steps leading to Eq. (1), and now find the temperature profile in the form

$$
\begin{aligned}
\frac{T(r)}{T_{R}}= & {\left[\frac{k(r)}{k_{R}}\right]^{3 / 5}\left[\frac{1+\delta_{R}}{1+\delta(r)}\right]^{2 / 5}\left\{1+\frac{2}{5} \frac{b_{R}}{1+\delta_{R}}\right.} \\
& \left.\times \int_{r}^{R} \frac{\mathrm{d} x}{x} \frac{v_{\mathrm{c}}^{2}(x)}{v_{R}^{2}}\left[\frac{k_{R}}{k(x)}\right]^{3 / 5}\left[\frac{1+\delta_{R}}{1+\delta(x)}\right]^{3 / 5}\right\},
\end{aligned}
$$

which extends Eq. (1) for $\delta>0$. Again, $n(r)$ is linked to $T(r)$ by $n=\left[k_{\mathrm{B}} T / k\right]^{3 / 2}$.

The actual temperature at the boundary is now lowered to $T_{R}=T_{2} /\left(1+\delta_{R}\right)$. This is seen from Rankine-Hugoniot conditions in the presence of turbulent pressure; the latter obviously implies the term $p_{2}\left(1+\delta_{R}\right)$ to be added on the righthand side of the stress balance, and the corresponding one $5 p_{2}\left(1+\delta_{R}\right) v_{2} / 2$ in the energy flow (see Eqs. (B1) in Lapi et al. 2005).

In our numerical computations that follow we adopt for fully developed turbulence the simple functional shape (rather than a constant $\delta$ as used by Bode et al. 2009)

$\delta(r)=\delta_{R} \mathrm{e}^{-(R-r)^{2} / \ell^{2}}$,

which decays on the scale $\ell$ inward of a round maximum, a smoothed-out representation of the inertial range. This is illustrated in Fig. 2 for three cases with $\ell=0.9 R, \ell=0.5 R$, and $\ell=0.2 R$. The runs $\delta(r)$ we adopt are consistent with those recently indicated by numerical simulations (e.g., Lau et al. 2009).

\section{Results}

We illustrate the resulting temperature and pressure profiles in Fig. 3. Relative to pure thermal HE with $\delta_{R}=0$, in the outskirts the thermal pressure lowers since it is helped by turbulent motions in sustaining the equilibrium, while the pressure run is moderately steeper. At the center, the pressure is mainly contributed by the thermal component.

It is seen that the variations in temperature (with the classic CC shape) are mild, and primarily stem from the reduction of $T_{R} / T_{2}$ by the factor $1+\delta_{R}=1.4$ discussed in Sect. 3 . Thus

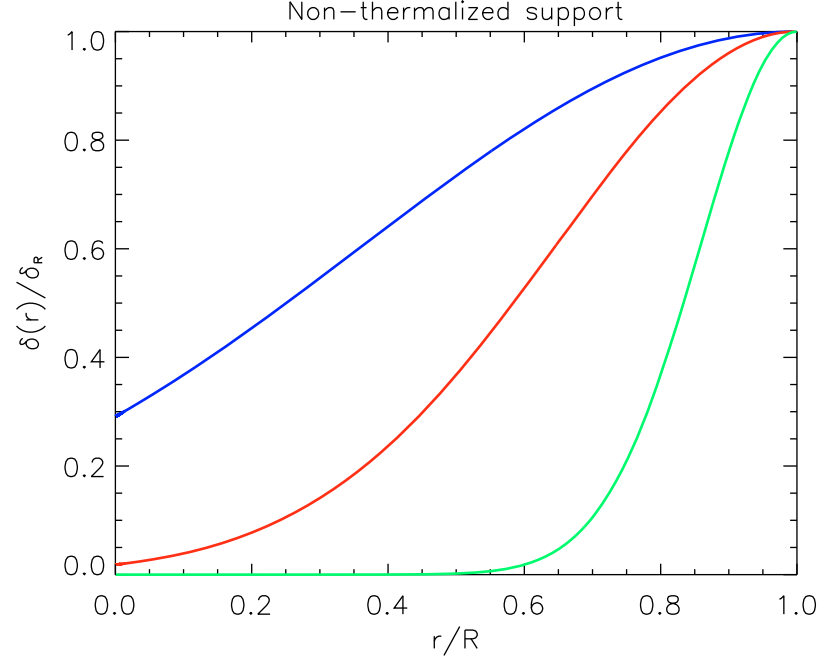

Fig. 2. Nonthermalized pressure support $\delta(r) \equiv p_{\text {nth }} / p_{\text {th }}$ normalized to the boundary value $\delta_{R}$ computed after Eq. (8). The blue line refers to $\ell=0.9 R$, red line to $\ell=0.5 R$, and green line to $\ell=0.2 R$.

we recognize the saturation or bending over of entropy on scales $r \lesssim R / 2$ (see Sect. 2) to constitute the primary cause of the steep temperature profiles observed by Suzaku. These drop by factors around 10 from the peak to the outer boundary in the low- $z$ clusters like A1795 and PKS0745-191.

This view is confirmed by Fig. 4, where we illustrate our best fits with the SM to the temperature and surface brightness profiles for the two clusters A1795 (north sector) and A1689 (azimutally averaged), one at a low and the other at a relatively high $z$. The figure shows that the SM with an entropy slope decreasing through the outskirts from standard values $a \approx 1.1$ at $r<R / 2$ to $a \approx-1.8$ at $R$ (just as expected in Sect. 2.2) fits the temperature profile of the low- $z$ cluster A1795 much better than the case with uniform slope $a \approx 1.1$. As expected, the difference is barely discernible for the relatively high- $z$ cluster A1689. Both fits are only mildly affected when including in the SM the turbulent support. The linked $n(r)$ profiles flatten out to enhanced brightness landings (cf. insets in Fig. 4), a simple warning of interesting temperature and turbulence distributions.

A far-reaching consequence of turbulent support shows up (see Fig. 5, left) in the reconstructions of DM masses from X-ray observables based on reversing the thermal HE equation (cf. Sarazin 1988, p. 92). It is seen that the mass reconstructed when ignoring the nonthermalized component deviates from the true mass by different amounts, and for low values of the dissipation scale $\ell$ may even have a non-monotonic behavior, not unlike the results by Kawaharada et al. (2010) in three sectors of A1689 (cf. their Fig. 8). In all cases, the reconstructed mass within $R$ is negatively biased by $20-30 \%$ relative to the true one. Accounting for such a bias constitutes a key point to resolve the tension between weak lensing and X-ray masses (e.g., Nagai et al. 2007; Lau et al. 2009; Meneghetti et al. 2010), and in deriving precise cosmological parameters from statistics of cluster masses via the fast X-ray observations (see Vikhlinin et al. 2009).

The above picture may be double-checked in individual clusters by directly gauging with the SZ scattering how the electron pressures are lowered in the presence of outer turbulence. In Fig. 5 (right) it is seen that along the line of sight running mainly into the outskirts, we expect the latter to be 20-30\% lower than the pure thermal case. This is stronger by a factor of about 5 than the effects from delayed equipartition between electron and ion 
A. Cavaliere et al.: Nonthermal support for the ICM
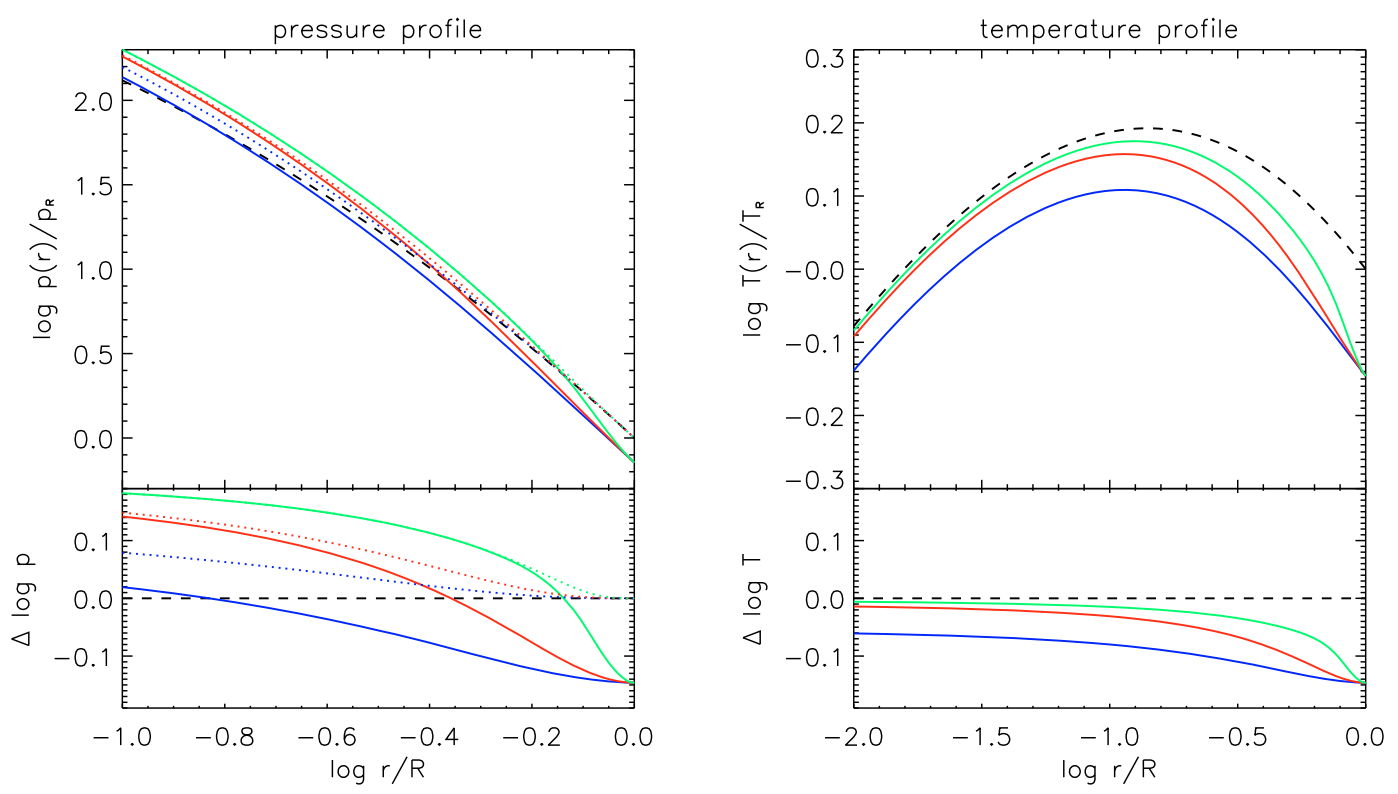

Fig. 3. Profiles of pressure and temperature computed with the SM. The dashed line illustrates the pure thermal (laminar) case, while solid lines illustrate the turbulent case with $\delta_{R}=40 \%$ and different $\ell$ (color code as in Fig. 2). In the left panel, solid lines refer to the thermal pressure, dotted ones to the total pressure.
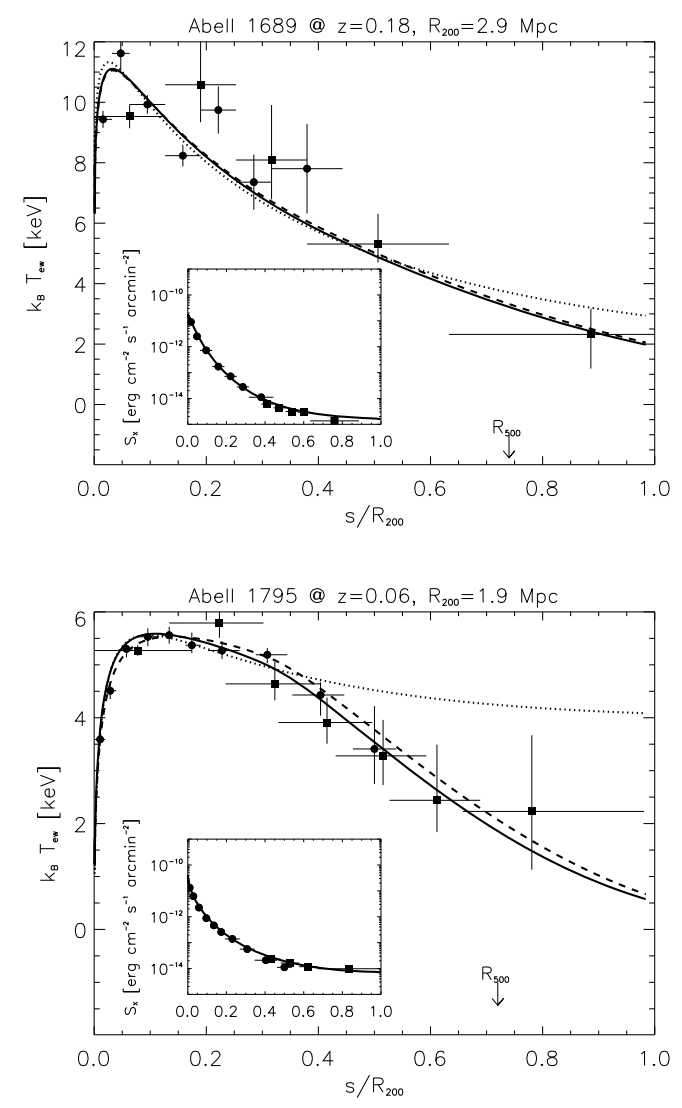

Fig. 4. Profiles of projected X-ray temperature (brightness in the insets) for the CC clusters A1689 (top) and A1795 (bottom). Data are from Snowden et al. (2008) with XMM-Newton (circles), and from Bautz et al. (2009) and Kawaharada et al. (2010) with Suzaku (squares). The solid lines represent our best fits with the SM extended to include turbulence after Eqs. (7) and (8) with $\delta_{R}=40 \%$ and $\ell=0.5 R$. The dashed ones illustrate the outcomes in the absence of turbulence, but still with entropy decreasing outwards as in Lapi et al. (2010). For comparison, the dotted lines illustrate the case with an uniform entropy slope. temperatures downstream of the shock over the relevant mean free path. A corresponding reduction is implied in the requirements for sensitivity and/or observation times with upcoming sophisticated instruments like ALMA (see Wong \& Sarazin 2009). Meanwhile, statistical evidence of SZ reductions has been extracted from stacked data by Komatsu et al. (2010).

\section{Discussion and conclusions}

To answer the issue raised at the end of Sect. 1, we investigated the connection of ICP turbulence in cluster outskirts with virial accretion shocks. Among the variety of shocks found in numerical simulations, these are most amenable to a simple treatment, which may also shed light on more complex conditions. A result to stress is the inverse nature of the connection that follows from combining the key Eqs. (4) and (6): as the Mach number $\mathcal{M}$ of the shock decreases, the postshock entropy $k_{2} \propto T_{2} / n_{2}^{2 / 3}$ lowers with $\mathcal{M}^{2}$, while the residual bulk energy $v_{2}^{2} / v_{1}^{2}$ rises with $\mathcal{M}^{-2}$. In other words, saturation of entropy production and increasing bulk flows to drive turbulence occur together.

To pinpoint when this is bound to occur, we base on the quantity $\dot{M} v_{1}^{2}\left(v_{2} / v_{1}\right)^{2} \propto d^{5 / 3} \epsilon^{-3}\left(v_{2} / v_{1}\right)^{2}$ including three factors: the infall speed $v_{1}^{2} \propto d^{2 / 3} / \epsilon^{2}$, see Eq. (3); the accretion rate $\dot{M} \propto d / \epsilon$; and the residual kinetic energy ratio $v_{2}^{2} / v_{1}^{2}$, see Eq. (6) and discussion thereafter. Such a quantity depends strongly on $d$ and even more on $\epsilon$, which render the effects on the outskirt's growth of the cosmological expansion accelerated by the dark energy, and of the declining shape of the initial DM perturbation wings, respectively.

This leads us to predict that the turbulent support starts to increase on average for $z<1 / 2$ during the late development of the cluster outskirts when $d \approx 1 / 2$ and $\epsilon>1$ apply. Then turbulence briskly rises at $z \lesssim 0.3$ when the shocks become transonic. On the other hand, environmental (even anisotropic) variance is introduced when the residual energy flow $\dot{M} v_{2}^{2}$, strongly dependent on $\epsilon$, is modulated by the adjacent filament/void structure. At very low $z$, a high level of thermal support will persist in sectors adjacent to filaments, as is apparently the case with A1795 

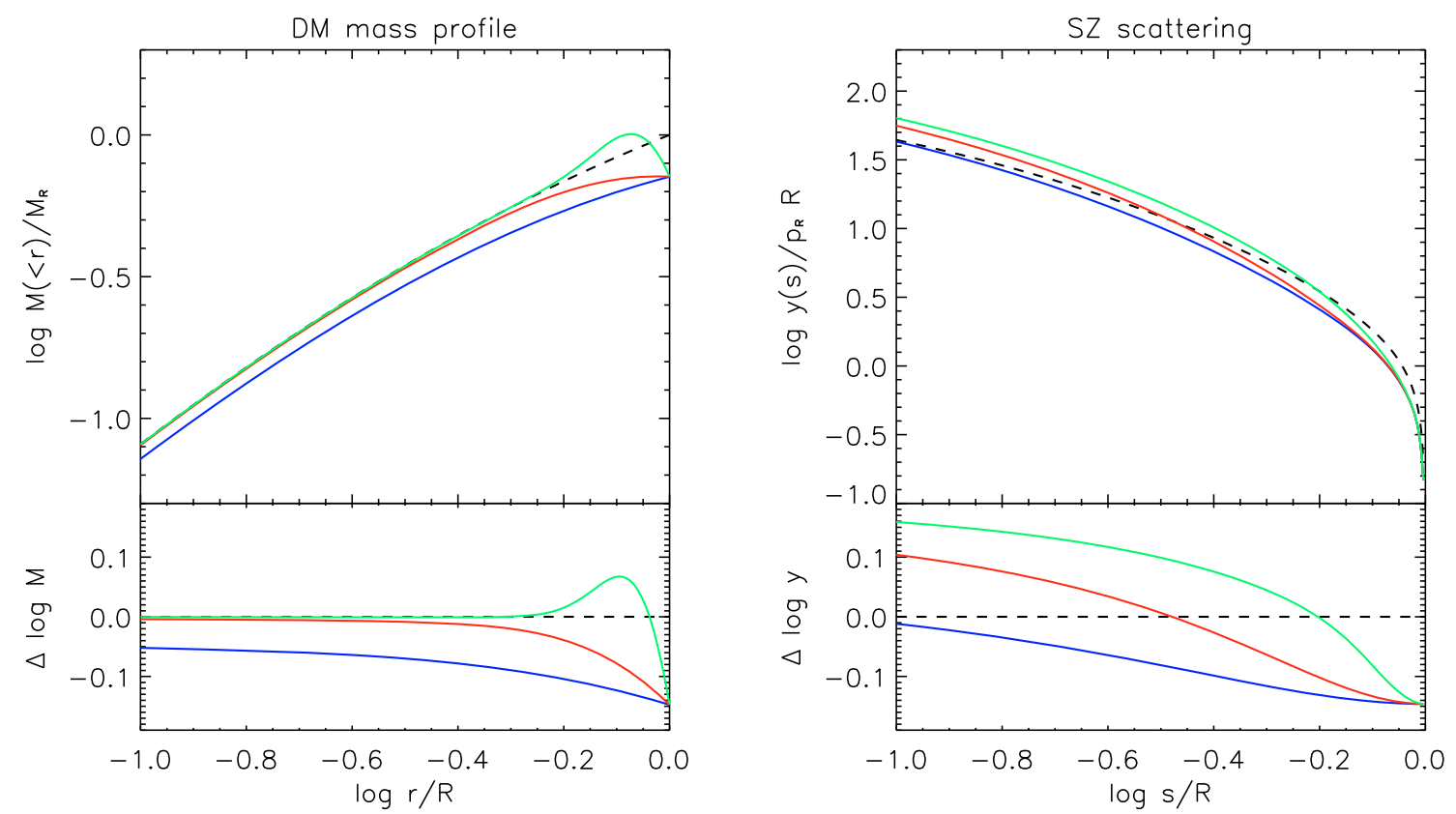

Fig. 5. Left: profile of DM mass. The dashed line illustrates the true mass, while the solid lines illustrate what is reconstructed from X-ray observables on assuming pure thermal HE. Right: projected profile of SZ scattering; the dashed line refers to the purely thermal case, while solid lines refer to the turbulent case. In both panels $\delta_{R}=40 \%$ and different values of $\ell$ (color code as in Fig. 2) are adopted.

and PKS0745-191 (see Bautz et al. 2009; George et al. 2009). By the same token, values $\epsilon>1$ will prevail in cluster sectors facing a void, causing an early onset of turbulence there given sufficient $\dot{M}$. Meanwhile, in neighboring sectors facing a filament, values $\epsilon \approx 1$ still hold and the thermal support may still prevail, a condition that apparently applies to A1689 (see Kawaharada et al. 2010; Molnar et al. 2010).

With the closer focus provided by the SM, we find that while the shocks weaken not only the boundary entropy production saturates but also the whole outer entropy distribution $k(r)$ flattens and the temperature profiles $T(r)$ become steeper, and do so consistently with the observations. Eventually, as the inflow approaches the transonic regime, $k(r)$ tends to bend over and $T(r)$ to steepen somewhat more.

But then subsonic turbulence arises at the boundary. Its inner distribution follows the classic picture of the turbulent motion fragmentation, with a scale for its final dissipation which is still debated. This demands closer probing, which we have tackled with the fast, analytic tool provided by the SM. The present data from joint X-ray and weak lensing observations (see Kawaharada et al. 2010; Molnar et al. 2010; Zhang et al. 2010) concur with simulations (see Lau et al. 2009) to show no evidence of a dissipation scale that is much shorter than $\sim 10^{2} \mathrm{kpc}$, conceivably set by tangling of the magnetic field (see discussions by Narayan \& Medvedev 2001; Brunetti \& Lazarian 2007).

To summarize, in the outskirts of relaxed clusters we find the following.

- Turbulence is related to weakening shocks at late cosmic epochs $z<0.3$, when saturation of entropy production causes steep temperature profiles.

- Turbulent excess pressures $\delta_{R}=p_{\text {nth }} / p_{\text {th }}$ up to $40 \%$ arise at the boundary, declining inwards on scales of $\ell \sim 100 \mathrm{kpc}$.

- The overall masses derived from X-rays are necessarily biased low, down to $20-30 \%$ when such a turbulent support is ignored.

These findings are consistent with the current observational and numerical data. Moreover, we predict that
- variance concerning steep $T(r)$ and turbulent support in cluster sectors will be correlated with the filament modulation of the adjacent environment,

- the SZ scattering will be considerably lowered relative to the pure thermal case, along the lines of sight running mainly into the outskirts or their sectors where X-rays concur with lensing data in signaling turbulent support.

We stress that the SM provides a fast tool for representing and probing the conditions of smooth inflows that prevail in the outskirts of relaxed CC clusters, away from mergers that scar the NCCs and constitute the realm of detailed but time-consuming numerical simulations.

A final comment concerns the giant radiohalos observed in the centers of several clusters to emit synchrotron radiation. These suggest that nonthermal support may be contributed by a mixture of magnetic field and relativistic particles accelerated by shocks and turbulence due to mergers (see Brunetti et al. 2007; Biermann et al. 2009; Brunetti et al. 2009). With their limited acceleration efficiency and short persistence, these processes are apparently widespread in NCC clusters, mainly at $z>0.2$, so they have minimal superposition or interference with the substantial, low- $z$, long-lived, outer turbulent component concerning mainly CCs, which we have addressed here.

The picture we pursue envisages the infall kinetic energy to thermalize along two channels. First, supersonic inflows achieve thermalization at the shock transition via a sharp jump (or a few jumps within a layer of limited thickness, see Lapi et al. 2005). Second, the subsonic turbulent motions left over downstream of the shocks, particularly downstream of weak shocks, are dispersed into a cascade of many effective degrees of freedom (see Landau \& Lifshitz 1959), down to scales where dissipation becomes effective. The channels' branching ratio shifts toward the latter when the shock weakens, for $z \lesssim 0.3$; meanwhile, turbulence concurs with thermal pressure to support the ICP equilibrium in the outskirts. The picture substantiates the following formal remark: Eq. (7) corresponds to Eq. (1) for the variable $T(1+\delta)$ in terms of the extended entropy $k(1+\delta)$. 
This picture of onset and development of outer ICP turbulence may be fruitful in other contexts, in particular for shocks and turbulence in wakes around mergers. Thus it warrants close modeling with our fast yet precise analytic SM, to probe the key turbulence features, amplitude and scale, over wide cluster samples in a range of $z$.

Acknowledgements. This work was supported by ASI and INAF. We thank our referee for helpful comments and suggestions. A.L. thanks SISSA and INAFOATS for their warm hospitality.

\section{References}

Bautz, M. W., Miller, E. D., Sanders, J. S., et al. 2009, PASJ, 61, 1117 Biermann, P. L., Becker, J. K., Caramete, L., et al. 2009, Nucl. Phys. Supp., 190, $61 \mathrm{~B}$

Binney, J. 1974, MNRAS, 168, 73

Birkinshaw, M., \& Lancaster, K. 2007, NewAR, 51, 346

Bode, P., Ostriker, J. P., \& Vikhlinin, A. 2009, ApJ, 700, 989

Bonafede, A., Feretti, L., Murgia, M., et al. 2010, A\&A, 513, A30

Brunetti, G., \& Lazarian, A. 2007, MNRAS, 378, 245

Brunetti, G., Venturi, T., Dallacasa, D., et al. 2007, ApJ, 670, L5

Brunetti, G., Cassano, R., Dolag, K., \& Setti, G. 2009, A\&A, 507, 661

Cavagnolo, K., Donahue, M., Voit, G. M., \& Sun, M. 2009, ApJS, 182, 12

Cavaliere, A., Lapi, A., \& Menci, N. 2002, ApJ, 581, L1

Cavaliere, A., Lapi, A., \& Fusco-Femiano, R. 2009, ApJ, 698, 580

Doroshkevich, A. G. 1973, SvA, 16, 986

Evrard, A. E. 1990, ApJ, 363, 349

Fakhouri, O., Ma, C.-P., \& Boylan-Kolchin, M. 2010, MNRAS, 406, 2267

Fusco-Femiano, R., Cavaliere, A., \& Lapi, A. 2009, ApJ, 705, 1019

Genel, S., Bouché, N., Naab, T., Sternberg, A., \& Genzel, R. 2010, ApJ, 719, 229

George, M. R., Fabian, A. C., Sanders, J. S., Young, A. J., \& Russell, H. R. 2009, MNRAS, 395, 657

Giacconi, R., et al. 2009, in Astro2010: The Astronomy and Astrophysics Decadal Survey (Science White Papers), 90

Govoni, F., Murgia, M., Feretti, L., et al. 2006, A\&A, 460, 425

Hoshino, A., Patrick Henry, J., Sato, K., et al. 2010, PASJ, 62, 371

Iapichino, L., \& Niemeyer, J. C. 2008, MNRAS, 388, 1089

Inogamov, N. A., \& Sunyaev, R. A. 2003, AstL, 29, 791

Kawaharada, M., Okabe, N., Umetsu, K., et al. 2010, ApJ, 714, 423

Kolmogorov, A. 1941, Dokl. Akad. Nauk SSSR, 30, 301

Komatsu, E., Smith, K. M., Dunkley, J., et al. 2010, ApJS, accepted [arXiv: 1001.4538]

Landau, L. D., \& Lifshitz, E. M. 1959, Fluid Mechanics (Oxford: Pergamon)

Lapi, A., \& Cavaliere, A. 2009, ApJ, 692, 174

Lapi, A., Cavaliere, A., \& Menci, N. 2005, ApJ, 619, 60
Lapi, A., Fusco-Femiano, R., \& Cavaliere, A. 2010, A\&A, 516, A34

Lau, E. T., Kravtsov, A. V., \& Nagai, D. 2009, ApJ, 705, 1129

Lu, Y., Mo, H. J., Katz, N., \& Weinberg, M. D. 2006, MNRAS, 368, 1931

Mahdavi, A., Hoekstra, H., Babul, A., \& Henry, J. P. 2008, MNRAS, 384, 1567

Markevitch, M., \& Vikhlinin, A. 2007, Phys. Rep., 443, 1

McCarthy, I. G., Bower, R. G., Balogh, M. L., et al. 2007, MNRAS, 376, 497

McNamara, B. R., \& Nulsen, P. E. J. 2007, ARA\&A, 45, 117

Meneghetti, M., Rasia, E., Merten, J., et al. 2010, A\&A, 514, A93

Molendi, S., \& Pizzolato, F. 2001, ApJ, 560, 194

Molnar, S. M., Chiu, I.-N., Umetsu, K., et al. 2010, ApJ, 724, L1

Monin, A. S., \& Yaglom, A. M. 1965, Statistical Hydromechanics (Nauka: Moscow)

Narayan, R., \& Medvedev, M. V. 2001, ApJ, 562, L129

Obukhov, A. M. 1941, Dokl. Akad. Nauk. SSSR, 1, 22

Nagai, D., Kravtsov, A. V., \& Vikhlinin, A. 2007, ApJ, 668, 1

Paul, S., Iapichino, L., Miniati, F., Bagchi, J., \& Mannheim, K. 2010, ApJ, submitted [arXiv: 1001.1170$]$

Pfrommer, C., \& Jones, T. W. 2010, ApJ, submitted [arXiv: 1004. 3540]

Pratt, G. W., Arnaud, M., Piffaretti, R., et al. 2010, A\&A, 511, A85

Reiprich, T. H., Hudson, D. S., Zhang, Y.-Y., et al. 2009, A\&A, 501, 899

Ricker, P. M., \& Sarazin, C. L. 2001, ApJ, 561, 621

Ryu, D., Kang, H., Hallman, E., \& Jones, T. W. 2003, ApJ, 593, 599

Ryu, D., Kang, H., Cho, J., Das, S., et al. 2008, Science, 320, 909

Sanders, J. F., Fabian, A. C., \& Smith, R. K. 2010, MNRAS, in press [arXiv: 1008.3500 ]

Sarazin, C. L. 1988, X-ray Emission from Clusters of Galaxies (Cambridge: Cambridge Univ. Press)

Schäfer, M., \& Bartelmann, M. 2007, MNRAS, 377, 253

Schücker, P., Finoguenov, A., Miniati, F., Böhringer, H., \& Briel, U. G. 2004, A\&A, 426, 387

Skillman, S. W., O’Shea, B. W., Hallman, E. J., Burns, J. O., \& Norman, M. L. 2008, ApJ, 689, 1063

Snowden, S. L., Mushotzky, R. F., Kuntz, K. D., \& Davis, D. S. 2008, A\&A, 478,615

Sunyaev, R. A., \& Zeldovich, Ya. B. 1972, A\&A, 20, 189

Tozzi, P., \& Norman, C. 2001, ApJ, 546, 63

Valageas, P., \& Silk, J. 1999, A\&A, 350, 725

Valdarnini, R. 2010, A\&A, in press [arXiv: 1010.3378]

Vazza, F., Brunetti, G., Gheller, C., \& Brunino, R. 2010, New Astron., 15, 695

Vikhlinin, A., Kravtsov, A. V., Burenin, R. A., et al. 2009, ApJ, 692, 1060

Voit, G. M. 2005, Rev. Mod. Phys., 77, 207

Voit, G. M., Balogh, M. L., Bower, R. G., et al. 2003, ApJ, 593, 272

Wang, J., Navarro, J. F., Frenk, C. S., et al. 2010, MNRAS, submitted [arXiv: 1008. 5114]

Weinberg, S. 2008, Cosmology (Oxford: Oxford Univ. Press)

Wong, K.-W., \& Sarazin, C. L. 2009, ApJ, 707, 1141

Wu, K. K. S., Fabian, A. C., \& Nulsen, P. E. J. 2000, MNRAS, 318, 889

Zhang, Y.-Y., Okabe, N., Finoguenov, A., et al. 2010, ApJ, 711, 1033

ZuHone, J. A., Markevitch, M., \& Johnson, R. E. 2010, ApJ, 717, 908 